\title{
Etnobotánica de las plantas más utilizadas en el Recinto Jauneche, Cantón Palenque, Provincia Los Ríos, Ecuador
}

\section{Ethnobotany of the most used plants in the Jauneche Enclosure, Palenque Canton, Los Ríos Province, Ecuador}

\section{Andrea Gabriela Valdiviezo ${ }^{1^{*}} \&$ Carmita Bonifaz ${ }^{2}$}

\author{
${ }^{1}$ Consultora independiente \\ 2 Universidad de Guayaquil, Herbario GUAY, Departamento de Botánica, Facultad \\ de Ciencias Naturales, Av. Raúl Gómez Lince s.n. y Av. Juan Tanca Marengo (campus \\ Mapasingue), Guayaquil-Ecuador
}

Recibido 3 agosto 2021; revisado 26 agosto 2021; aceptado 20 de diciembre 2021; disponible en línea 30 de diciembre 2021.

\begin{abstract}
Resumen
El estudio de las plantas más utilizadas en el Recinto Jauneche, Cantón Palenque, Provincia Los Ríos, Ecuador, tuvo como finalidad conocer la etnobotánica local. Se procedió a realizar un levantamiento de información primaria donde se entrevistaron a tres personas con conocimiento sobre plantas útiles, con los datos de la fase inicial se realizaron encuestas simples a 20 personas de 35 años en adelante, se registró un total de 84 plantas útiles. Una vez obtenida la información se realizó la respectiva clasificación etnobotánica registrando los siguientes usos: medicinal, alimenticia, construcción y rudimentaria (utensilios caseros). Las plantas medicinales fueron las de mayor representatividad con un 57\%, seguida por las alimenticias con un $26 \%$, construcción $13 \%$ y rudimentaria $4 \%$, con la recolección de muestras se realizó la identificación taxonómica donde se encontraron 37 familias y 61 géneros, la familia con mayor número de especies fueron Lamiaceae y Asteraceae. Estos resultados indican que el uso plantas útiles en el Recinto Jauneche puede ser considerado alto, debido a que se registró información con una muestra de 23 personas solamente, indicando que el conocimiento etnobotánico está presente en los habitantes del recinto Jauneche, aduciendo este factor a la relación cercana que mantienen con el Bosque de la Estación Biológica Pedro Franco Dávila.
\end{abstract}

\begin{abstract}
The study of the most used plants in the Jauneche Enclosure, Palenque Canton, Los Ríos Province, Ecuador, had the goal of knowing the local ethnobotany. To obtain the primary information three people with knowledge about useful plants were interviewed, with the data of the initial phase simple surveys were carried out on 20 people over 35 years old, a total of 84 useful plants were recorded. Once the information was obtained, the respective ethnobotanical classification was carried out, recording the following uses: medicinal, food, construction and rudimentary (homemade utensils). Medicinal plants were the most representative with $57 \%$, followed by food plants with $26 \%$, construction $13 \%$ and rudimentary $4 \%$, the taxonomic identification based on collected samples yielded 37 families and 61 genera, the family with the largest number of species were Lamiaceae and Asteraceae. These results indicate that the use of useful plants in the Jauneche Enclosure could be regarded as high, because the gathered information was obtained from 23 people only, indicating that ethnobotanical knowledge is present in the inhabitants of the Jauneche enclosure, adducing this factor to the close relationship they maintain with the forest of the Pedro Franco Dávila Biological Station.

Keywords: ancestral knowledge, coast, useful plants, vegetal resources.
\end{abstract}


Rev. Cient. Cien. Nat. Ambien. 15(2):278-288 Diciembre 2021
Valdiviezo \& Bonifaz • Etnobotánica de las plantas más utilizadas en el Recinto Jauneche, Cantón Palenque, Provincia Los Ríos, Ecuador

\section{Introducción}

La etnobotánica es la ciencia que tiene como finalidad el estudio de las plantas y su entorno social, es decir, su relación con las personas; las bases de ésta están ligadas a comunidades primitivas que lograron transmitir sus conocimientos de las especies vegetales útiles para el ser humano (Pardo, 2008). El término “etnobotánica" comenzó a utilizarse en 1895, aunque el conocimiento ancestral de las plantas útiles ha sido parte de muchas culturas existentes, desde las primeras civilizaciones (Hórak et al., 2015).

También cabe recalcar que el estudio de plantas útiles está estrechamente ligado a la etnobotánica la cual se encarga del estudio de las especies vegetales y su relación con los saberes ancestrales (Pardo \& Gómez, 2003). Este estudio científico ha tenido como resultado datos de las primeras civilizaciones, las cuales aprendieron a clasificar cada planta según su utilidad y aquellas que no eran beneficiosas para la comunidad (Hórak et al., 2015).

Además, los recursos vegetales son parte de la economía de diversas culturas ancestrales, los cuales han sabido aprovechar sus conocimientos en prácticas tradicionales sin alterar el ecosistema; estos conocimientos están ligados a la soberanía alimentaria de los pueblos y nacionalidades, fomentando el cuidado de los servicios ecosistémicos con los que están relacionados (Lagos et al., 2011).

Por lo tanto, las plantas útiles pueden estar clasificadas según el grupo étnico donde se encuentran, algunas de las especies pueden llegar a ser multiusos, pero la clasificación más común entre las especies vegetales útiles es: medicinales, alimenticias, ornamentales, artesanales, forrajeras, ceremoniales, toxicas, rudimentarias, industriales y construcción, entre otras (Del Vitto \& Petenatti, 2015).

El conocimiento tradicional sobre las plantas útiles es de vital importancia para la conservación de especies nativas y endémicas, también proteger la información existente sobre las especies vegetales permitirá que nuevas investigaciones científicas descubran plantas para elaborar nuevas medicinas, al mismo tiempo que se protege las áreas naturales (Ríos et al., 2008).

La pérdida del conocimiento tradicional o de los saberes ancestrales también afecta de manera directa o indirecta la economía de los pueblos originarios, en especial de aquellos que se sustentan de los productos que provee la naturaleza (Sánchez \& Torres, 2020).

Los estudios sobre plantas útiles realizados en Ecuador han sido de gran importancia porque han permitido definir las especies que han sido tradicionalmente utilizadas por pueblos originarios (de la Torre et al., 2008). Este conocimiento tradicional o ancestral de la flora ecuatoriana ha sido el resultado de importantes trabajos que se han realizado en la región costa, como lo es la flora útil del Bosque Protector Cerro Blanco Guayas-Ecuador (Cerón, 2002), así como el uso de la etnobotánica en enfermedades de la infancia por la etnia Chachi en Esmeraldas (Velásquez et al., 2016).

Por otro lado, en la costa ecuatoriana a inicios del siglo XX, diversos botánicos realizaron sus estudios en los pueblos originarios existentes donde obtuvieron conocimiento sobre plantas útiles, aunque en los bosques secos de la región costa la diversidad es relativamente baja, su endemismo es alto, también se toma en consideración la fragilidad de este tipo de ecosistemas, por lo que se ha considerado importante implementar nuevos planes de conservación (Huamantupa et al., 2010). Por otra parte, cabe recalcar que el uso de las plantas depende de las diferentes regiones del país (Álvarez, 2012).

Los grupos étnicos en el Ecuador se han caracterizado por tener un amplio uso de la flora que se encuentran en la selva, bosques u otros tipos de ecosistemas, esto es lo que motiva a las comunidades ancestrales a mantener intacta la flora existente, respetando el hábitat donde se encuentra, consumiendo solo lo necesario, esta relación que existe entre el hombre y naturaleza es lo que permite crear nuevas áreas naturales protegidas en comunidades locales (Polanco et al., 2018).

Sin embargo, la diversidad florística del Ecuador se encuentra en peligro por la pérdida o degradación de hábitats que pueden estar relacionados con factores antropogénicos o naturales (Imbaquingo, 2012). Por lo tanto, tomando en consideración la relación que existe entre un ecosistema y la sociedad, podemos decir que la pérdida del conocimiento tradicional sobre plantas útiles también influye en las áreas naturales protegidas (García et al., 2008).

Uno de los retos que siempre se ha presentado en la conservación de áreas naturales cercanas a centros poblados, es cuando en la comunidad local existe una creciente actividad agrícola basada en monocultivos o en algunos casos la práctica de ganadería, estas actividades van reduciendo espacios de áreas naturales ocasionando perdida de bosques donde existe una gran diversidad biológica (Aranguren, 2005).

Los trabajos etnobotánicos realizados en el Ecuador han dado a conocer un total de 5.172 plantas útiles (De la Torre, Navarrete, Macía \& Balslev, 2008) a pesar que estos estudios tienen mayor énfasis en la amazonia ecuatoriana existen investigaciones realizadas en las regiones de la Sierra y Costa. Yánez (2017) realizó un listado de 68 especies vegetales entre nativas e introducidas que son utilizadas en las zonas rurales de la región amazónica centro occidental, Napo, Pastaza y Morona Santiago, estas zonas rurales se caracterizan por poseer una población equilibrada entre indígenas y colonos; Cuchisacagua (2019), por el contrario reportó un total de 140 plantas útiles en la 
Rev. Cient. Cien. Nat. Ambien. 15(2):278-288

Diciembre 2021
Valdiviezo \& Bonifaz • Etnobotánica de las plantas más utilizadas en el Recinto Jauneche, Cantón Palenque, Provincia Los Ríos, Ecuador comunidad amazónica Verde Sumako perteneciente a la provincia de Orellana, las cuales fueron localizadas en fincas cercanas a la selva; Peña, Guerrero, Torre \& Sánchez (2020), reportaron en otra comunidad kichwa amazónica de la Provincia de Sucumbíos un total de 23 plantas vasculares útiles.

En la Sierra, Polanco, Vernaza \& Burbano (2018) reportan un total de 116 especies de plantas útiles que son vendidas en tres mercados de la ciudad de Quito, las cuales son alimenticias, medicinales, entre otros usos; Rodriguez, Narvaez \& Tuz (2020) reportan un total de 90 especies vegetales que son utilizadas por yerbateras quiteñas para realizar rituales, de estas plantas 43 presentan otros usos como condimento o rudimentarias; estas plantas tienen una gran importancia económica para las mujeres mayores que se dedican a este negocio. Sánchez \& Torres (2020) realizaron un estudio en la Provincia de Cañar, donde se registraron un total de 71 especies que se consideran útiles por los habitantes de la parroquia Taday, en el cantón Azogues.

En la región costa los estudios etnobotánicos o de plantas útiles son dirigidos a usos específicos, Velásquez et al. (2016), realizaron un estudio en el grupo étnico Chachi de la provincia de Esmeraldas sobre plantas medicinales que pueden curar enfermedades eruptivas las cuales son comunes durante la etapa infantil, registrando el uso de tres especies diferentes de plantas.

Zambrano, Buenaño \& Jiménez (2015), realizaron un estudio etnobotánico de plantas medicinales en la Parroquia San Carlos, Quevedo, Ecuador, donde registraron un total de 43 especies vegetales que son utilizadas como medicinales para aliviar distintas enfermedades. Por otra parte, Paredes, Buenaño \& Mancera (2015) reportaron un total de 33 plantas medicinales que son utilizadas por los pobladores de la comunidad San Jacinto en el Cantón Ventanas, en la Provincia de Los Ríos, Ecuador.

En las comunidades rurales se pueden encontrar especies vegetales que presentan o tienen mas de una utilidad, Rosero, Zambrano, García \& Viracocha (2020) realizaron una investigación sobre el culantro de monte (Eryngium foetidum L.) en la comunidad San Antonio de Padua del Cantón Quinsaloma, Provincia de los Ríos, Ecuador, donde encontraron que esta especie es utilizada como condimiento para alimentos y también como planta medicinal. Bonifaz (1997) realizó un estudio de plantas tóxicas en la hacienda La Juanita y en el Recinto Jauneche, donde se reportó un total de 26 especies vegetales tóxicas y de otras utilidades como ornamental, medicinal y/o alimenticia.

En la provincia de Los Ríos, específicamente en el Recinto Jauneche, se han encontrado plantas que tienen diferentes usos, esto va a depender de la parte que es empleada o de la etapa de crecimiento en que se encuentre, es decir, que una misma especie puede ser medicinal y tóxica (Bonifaz, 1997), por lo cual, es de vital importancia comprender el uso que se le dan a las especies vegetales y su relación con el bosque nativo, por lo cual se ha desarrollado la presente investigación que tiene como objetivo, conocer la etnobotánica de las plantas más utilizadas en esta área, lo que contribuirá al desarrollo de planes conservación y manejo que involucren a la comunidad local.

\section{Materiales y métodos}

\section{Área de estudio}

El recinto Jauneche está ubicado en el Cantón Palenque de la Provincia de los Ríos, sus límites son el cantón Mocache al Norte, Palenque al sur, al este Ventanas y al oeste Balzar (Figura 1). Este recinto se encuentra a una altura promedio de $70 \mathrm{msnm}$, donde durante la época lluviosa la precipitación promedio anual es de $1800-2000 \mathrm{~mm}$, con la presencia de un bosque tropical semideciduo correspondiente al Bosque Protector de la Estación Pedro Franco Dávila (Salazar, 2019).

\section{Obtención de datos}

El presente trabajo se lo realizó con el levantamiento de información primaria durante el mes de Julio del 2021, la primera fase consistió en entrevistar a tres personas con conocimiento sobre plantas y sus respectivos usos donde no se tomó en consideración la edad de los entrevistados por su amplio conocimiento en plantas útiles.

Con los datos obtenidos de los informantes claves, se procedió a verificar en la zona de estudio, en donde se reconoció dos sectores: las viviendas cercanas al bosque protector y aquellas que se encontraban cruzando la carretera principal. Como segunda etapa de levantamiento de información, se visitaron un total de 56 viviendas escogidas al azar de la zona céntrica del recinto Jauneche, se realizaron 4 a 5 visitas por día durante el mes de Julio del 2021.

\section{Generalización de las encuestas}

De acuerdo con los datos previamente obtenidos se procedió a encuestar a 20 personas de 45 años en adelante. El formato que se utilizó fue elaborado en una hoja de Microsoft Excel, con la respectiva información del informante, nombre, edad, sexo, ocupación, tiempo de residencia y grado de escolaridad, utilizando un modelo de encuesta de tipo abierta con los siguientes datos: plantas que utilizan, tipo de uso, uso específico y partes que se usan.

\section{Clasificación y procesamiento de datos etnobotánicos}

La información obtenida por medio de las encuestas y entrevistas a las personas seleccionadas fue ordenada en una base de datos empleando una hoja de cálculo en Microsoft Excel. 

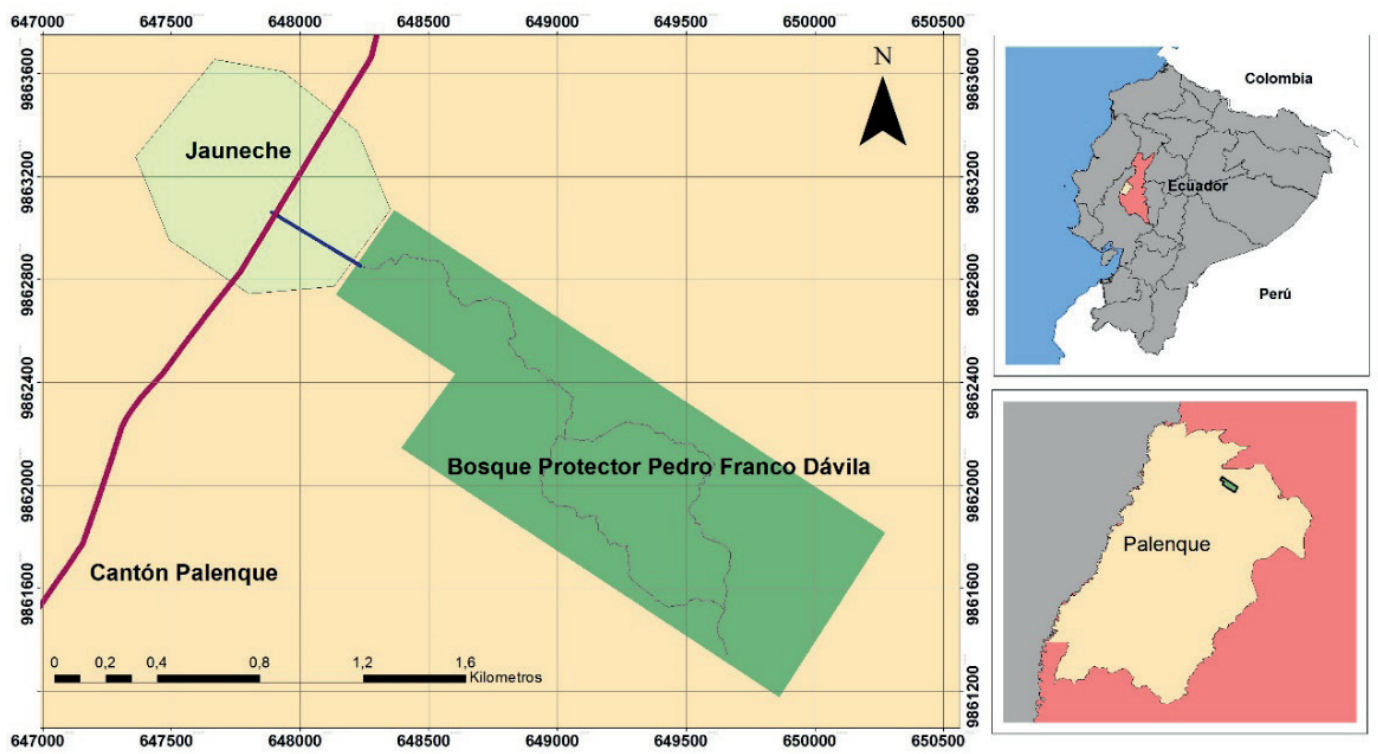

Leyenda

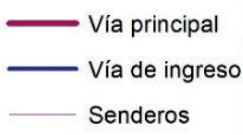

Figura 1. Mapa de ubicación del recinto Jauneche.

Recolección e identificación del material botánico Las muestras de cada planta útil fueron herborizadas y procesadas en el herbario de la Facultad de Ciencias Naturales de la Universidad de Guayaquil GUAY. La identificación taxonómica se realizó con el uso de la Flora de Jauneche con la ayuda de material fotográfico, usando la aplicación Pl@ntnet.

\section{Resultados}

Clasificación etnobotánica de las plantas más utilizadas del recinto Jauneche

De un total de 84 plantas que son utilizadas en el recinto Jauneche, $57 \%$ corresponden a uso medicinal, seguido por alimenticias con un $27 \%$, construcción con $13 \%$, mientras que las de uso rudimentario poseen la menor presencia con un $4 \%$, siendo el tipo de uso poco frecuente entre los informantes (fig. 2).

De un total de 23 entrevistados, se obtuvo la información de 84 plantas que son utilizadas en el Recinto Jauneche, cada planta fue registrada con su nombre local correspondiente, uso, hábito y partes que se usa (Anexo1).

La información etnobotánica levantada ha permitido clasificarlas como: plantas más utilizadas, plantas menos utilizadas, hábito de las plantas más utilizadas, partes utilizadas, uso específico y forma de preparación.

\section{Plantas más utilizadas}

Las especies que fueron nombradas de forma más frecuente correspondió al llantén con un 72\% (fig. 3), seguida por el oreganito con un $68 \%$, el mismo que es usado como condimento y la fruta de pan con un 64\% como alimento, las demás plantas son utilizadas en un $10 \%$ (fig. 4 ).

\section{Plantas menos utilizadas}

En un total de 59 plantas fueron mencionadas de uso poco frecuente con un $5 \%$, entre las que se encuentran tiatina (fig. 5), pedorrera, ruda de gallinazo, alivia dolor y ajenjo, utilizadas de 1 a 4 personas (fig. 6).

Hábito de las plantas más utilizadas en Jauneche En el uso de las plantas útiles es importante conocer el hábito de cada una de ellas. En el recinto Jauneche,

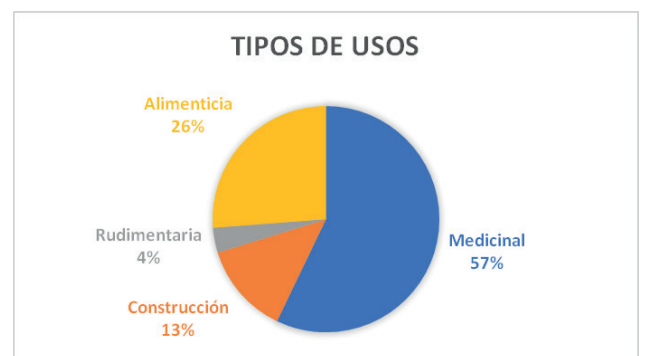

Figura 2. Porcentaje de plantas según el tipo de uso. 
Rev. Cient. Cien. Nat. Ambien. 15(2):278-288

Diciembre 2021
Valdiviezo \& Bonifaz • Etnobotánica de las plantas más utilizadas en el Recinto Jauneche, Cantón Palenque, Provincia Los Ríos, Ecuador según las encuestas realizadas el 53\% de plantas útiles son herbáceas, mientras que el $32 \%$ son árboles, los arbustos en un $14 \%$ y en el caso de las lianas o trepadoras un $1 \%$ (figura 7 ).

\section{Partes utilizadas}

De las plantas más utilizadas, las hojas son las más frecuentes con $47 \%$, seguidas por el fruto con un $24 \%$, $12 \%$ para el tronco de árboles, en algunos casos se utiliza toda la planta demostrada en el $11 \%$, corteza y látex con el $2 \%$, raíz y tubérculo con el $1 \%$ (fig. 8).

\section{Uso específico}

Las categorías de usos de las plantas presentaron mayor porcentaje aquellas que son utilizadas como comestibles con un $20 \%$, mientras que las maderables un $7 \%$. Un uso específico en las medicinales que presentaron un $7 \%$ fueron las plantas utilizadas en Baño medicinal, las plantas utilizadas para dolores estomacales se hicieron presentes con un 4\% (fig. 9).

\section{Forma de preparación}

En la forma de preparación de las plantas útiles se toma en consideración las alimenticias y medicinales, la infusión fue el método de preparación más común con $50 \%$, cocción con $30 \%$, emplasto $13 \%$ y aplicación directa $1 \%$ (fig. 10).

Identificación taxonómica de las plantas más utilizadas del Recinto Jauneche

La identificación taxonómica de las 84 plantas útiles dio como resultado una composición de 37 familias y 61 géneros (Anexo 2).

\section{Familias}

De las 37 familias presentes la más representativa es Lamiaceae con 8 especies, seguida por Asteraceae con 5 especies, Acanthaceae, Fabaceae, Malvaceae, Solanaceae con 4 especies, Euphorbiaceae, Moraceae, Plantaginaceae con 3 especies de plantas útiles, las familias restantes tuvieron de 1 a 2 especies (fig. 11).

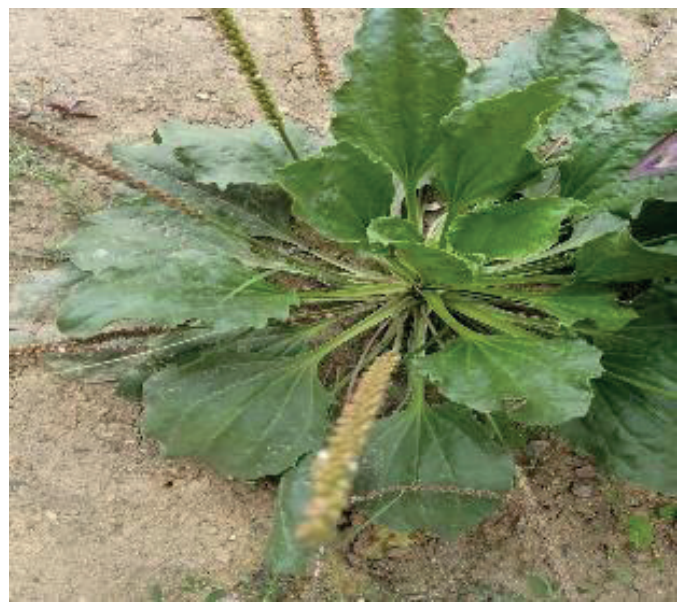

Figura 3. Planta más utilizada, Llantén (Plantago major).

\section{Géneros}

De los 61 géneros que se identificaron Inga tuvo mayor representatividad con 4 especies, seguido por Justicia con 3 especies, los géneros Cordia, Cymbopogon, Kalanchoe, Mentha, Ocimun y Solanum con 2 especies, los géneros restantes tuvieron solo una especie representante (fig. 12).

\section{Especies}

De las 84 plantas que se identificaron la especie con mayor uso por persona fue Plantago major con 18, seguida por Origanum majorana 17, Artocarpus communis 16, Dysphania ambrosioides 15, Cymbopogon citratus 14, Mentha piperita 13, Cymbopogon flexuosus 12 , las especies restantes fueron mencionadas por menos de 11 personas (fig. 13).

Datos de informantes etnobotánicos de las plantas más utilizadas en Jauneche

Las edades tomadas en consideración en las encuestas fueron de 35 a 90 años, las predominantes fueron de 56 a 65 años. El tiempo de residencia de los informantes con mayor porcentaje fueron los rangos de 20-30, 31$40,51-60$ años, mientras que el $17 \%$ fue representado por aquellos que tenían de 41-50 y 61-70 tiempo de residencia en el recinto. Entre los tipos de ocupación que se mencionaron están ama de casa y agricultores con un $33 \%$ cada uno, curandero y parteros con un $19 \%$, mientras que comerciante, carpintero y docente con un $5 \%$ demostrando la menor presencia en estas últimas ocupaciones.

Con respecto a la educación 10 personas tuvieron un grado de formación académica primaria, seguidos por los que no tuvieron ningún estudio académico con un total de 8 , mientras que los que estudiaron bachillerato fue de un 3 y título de pregrado uno, de las 23 personas entrevistadas y encuestadas el $53 \%$ pertenecen al género femenino, mientras que el género masculino tuvo una representación del $43 \%$ (Tabla 1, fig. 14).

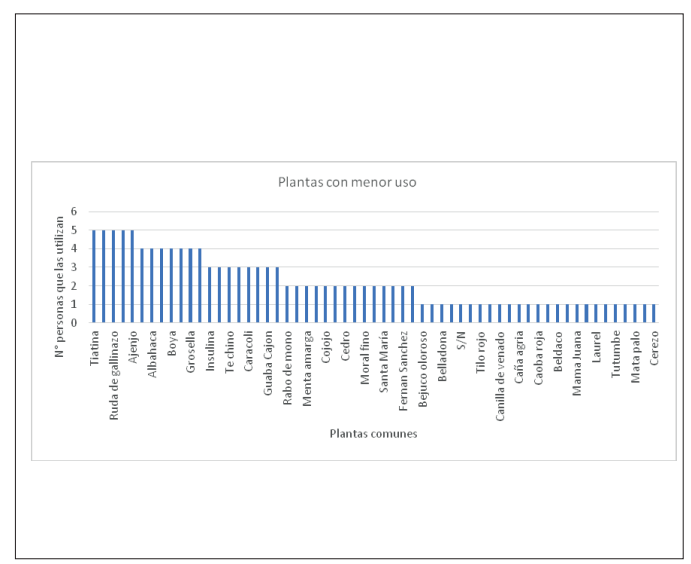

Figura 4. Listado de especies vegetales según el uso por persona. 
Rev. Cient. Cien. Nat. Ambien. 15(2):278-288 Diciembre 2021
Valdiviezo \& Bonifaz • Etnobotánica de las plantas más utilizadas en el Recinto Jauneche, Cantón Palenque, Provincia Los Ríos, Ecuador

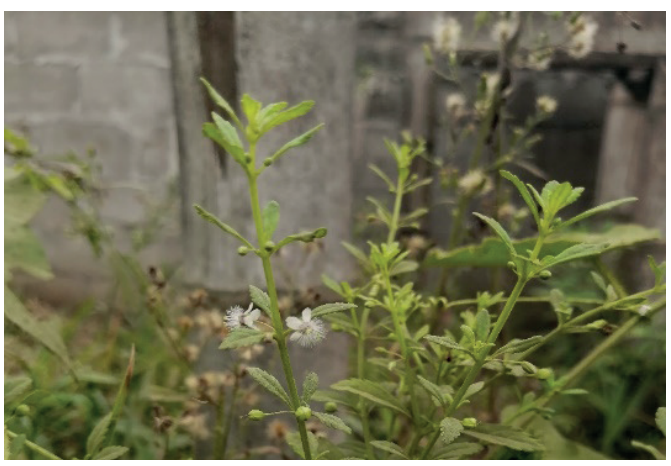

Figura 5. Tiatina (Scoparia dulcis) planta con menor uso.

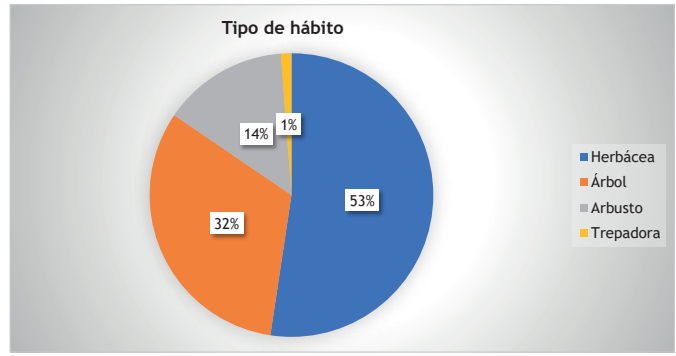

Figura 7. Hábito de las especies de plantas más utilizadas en Jauneche.

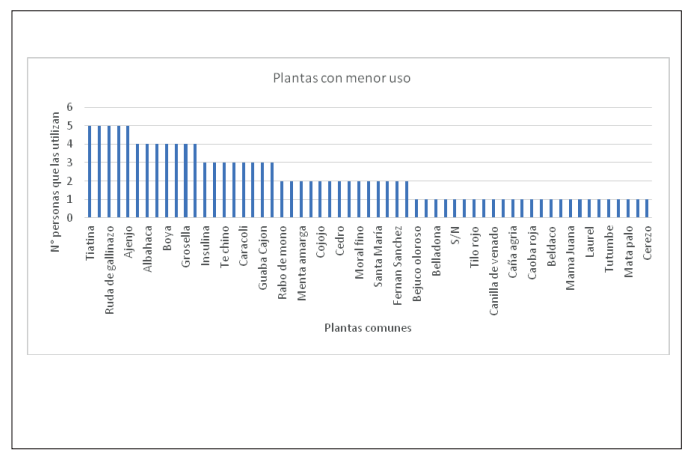

Figura 6. Listado de especies vegetales según el uso por personas.

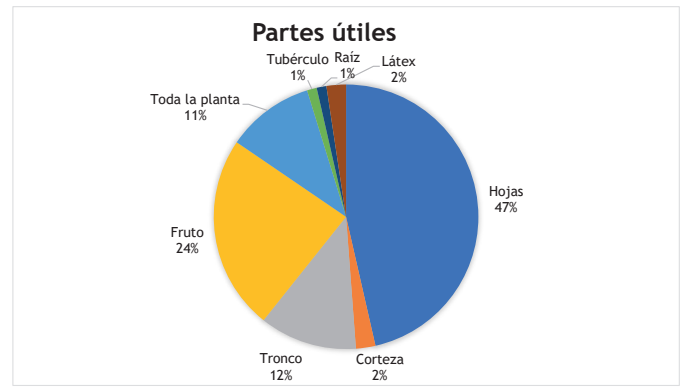

Figura 8. Partes más utilizadas de las plantas útiles.

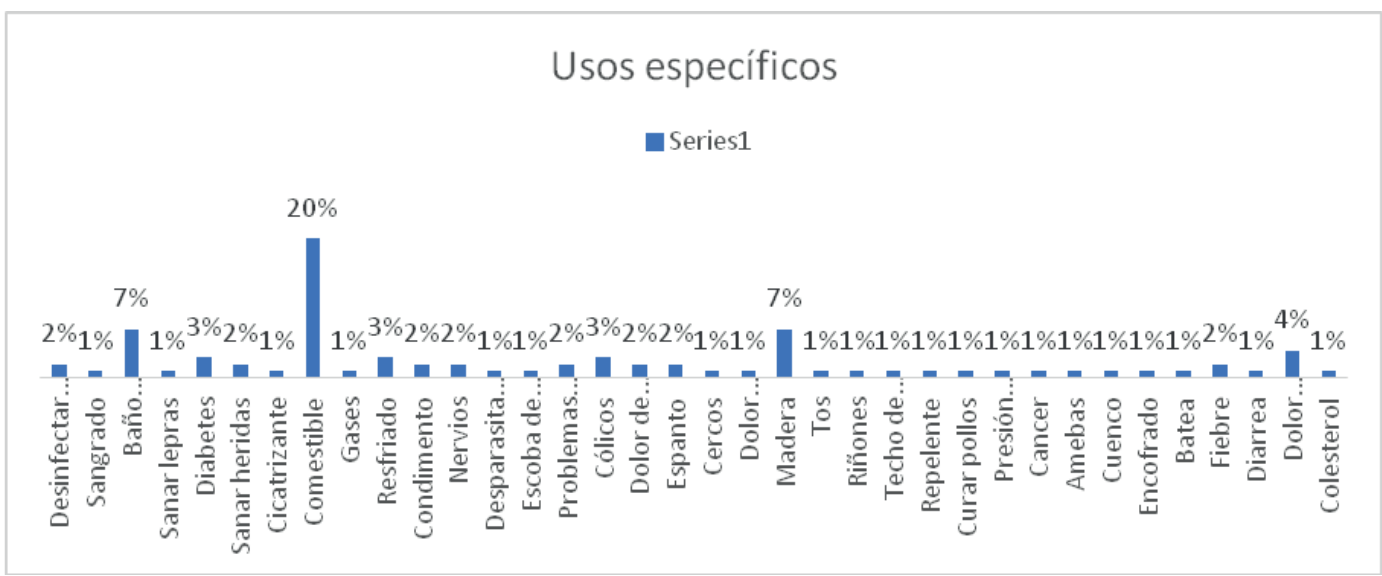

Figura 9. Categorías de usos de las plantas más utilizadas del recinto Jauneche. 
Rev. Cient. Cien. Nat. Ambien. 15(2):278-288
Valdiviezo \& Bonifaz • Etnobotánica de las plantas más utilizadas en el Recinto Jauneche, Cantón Palenque, Provincia Los Ríos, Ecuador

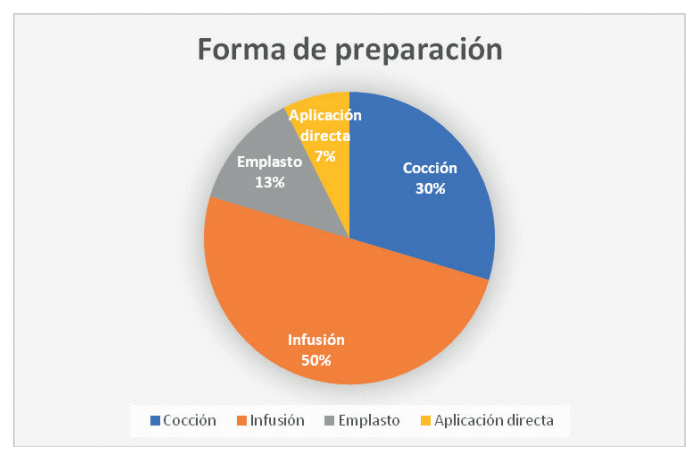

Figura 10. Formas de preparación de las plantas útiles.

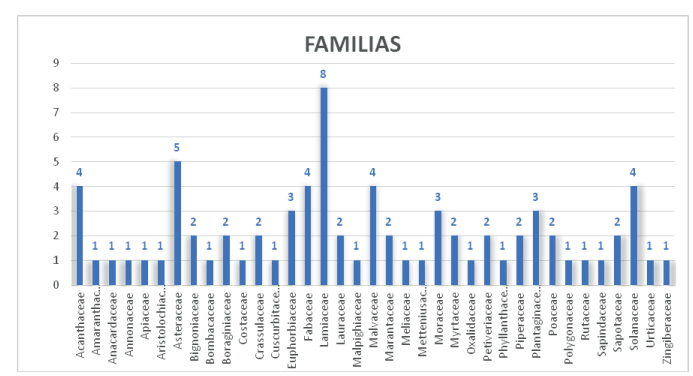

Figura 11. Familias de plantas útiles presentes en Jauneche.

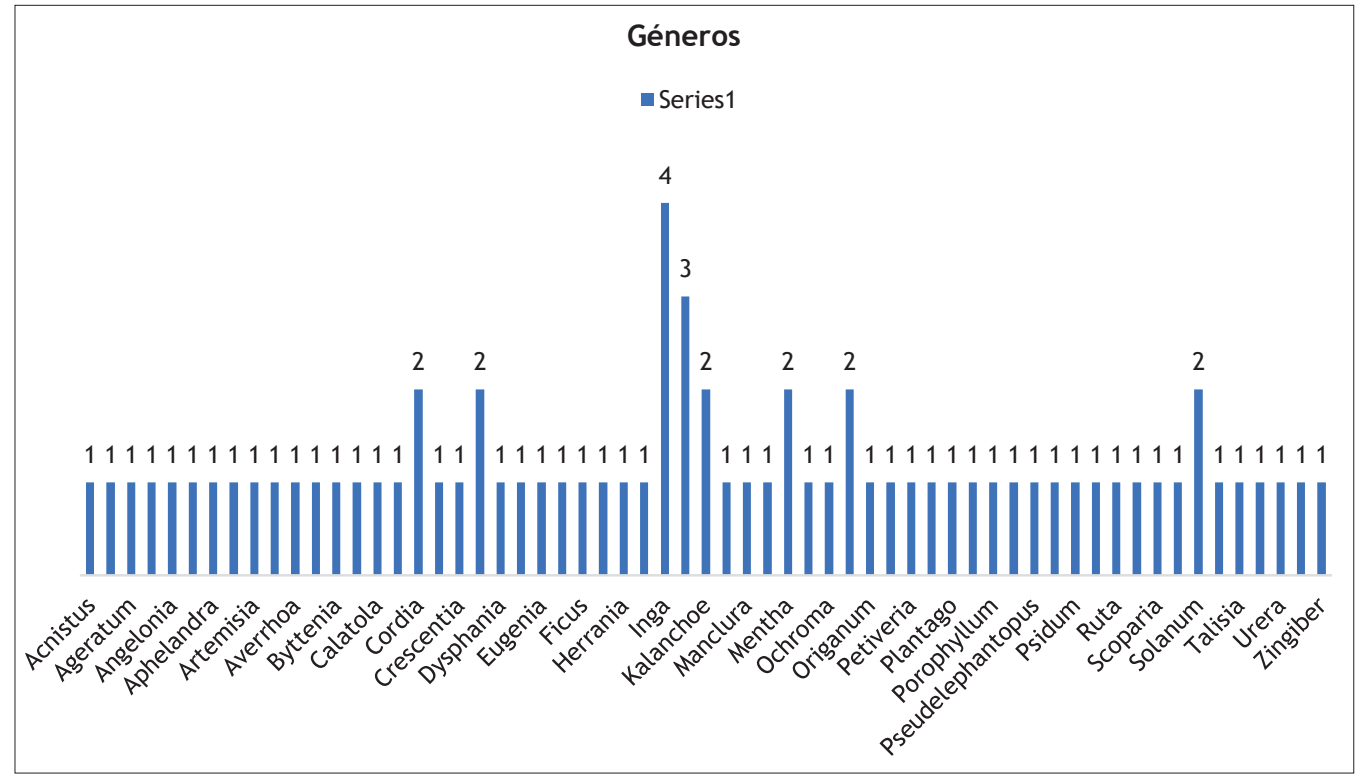

Figura 12. Géneros de plantas útiles presentes en Jauneche.

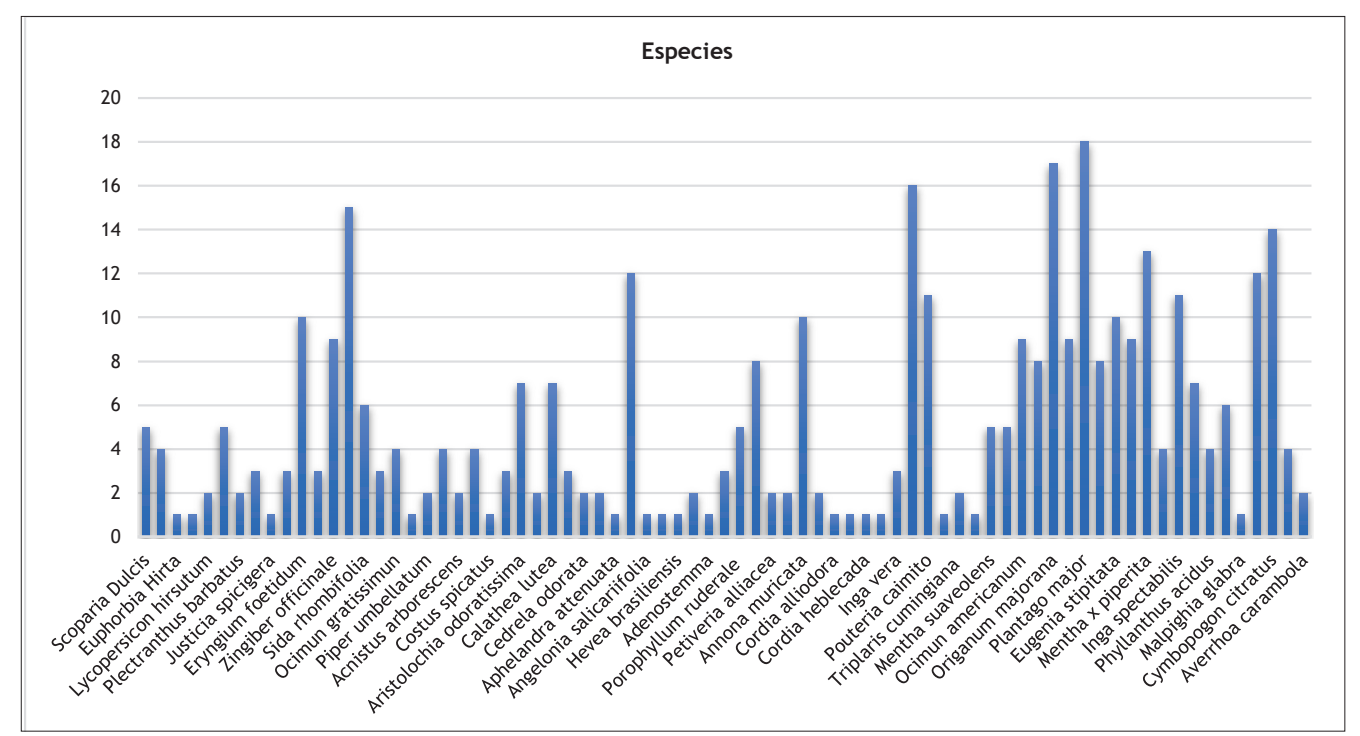

Figura 13. Frecuencia de uso medicinal de las especies de plantas útiles de Jauneche. 
Rev. Cient. Cien. Nat. Ambien. 15(2):278-288 Diciembre 2021
Valdiviezo \& Bonifaz • Etnobotánica de las plantas más utilizadas en el Recinto Jauneche, Cantón Palenque, Provincia Los Ríos, Ecuador

Tabla 1. Datos de los informantes que participaron en las encuestas y entrevistas.

\begin{tabular}{|c|c|c|c|c|c|c|c|c|c|}
\hline \multirow{2}{*}{\multicolumn{2}{|c|}{$\begin{array}{c}\text { Edad } \\
\text { Mínimo: } 37\end{array}$}} & \multirow{2}{*}{\multicolumn{2}{|c|}{$\begin{array}{l}\text { Tiempo de } \\
\text { residencia }\end{array}$}} & \multicolumn{2}{|c|}{ Ocupación } & \multicolumn{2}{|c|}{ Educación } & \multicolumn{2}{|c|}{ Género } \\
\hline & & & & Agricultor & 7 & Ninguna: & 8 & Hombre & Mujer \\
\hline \multicolumn{2}{|c|}{ Máximo: 86} & $20-30$ & 5 & Docente & 1 & Primaria: & 10 & $57 \%$ & $43 \%$ \\
\hline \multicolumn{2}{|c|}{ Amplitud:10 } & $31-40$ & 5 & Ama de casa & 7 & Bachillerato: & 3 & & \\
\hline $35-45$ & 1 & $41-50$ & 4 & Carpintero & 1 & Pre-grado: & 1 & & \\
\hline $46-55$ & 5 & $51-60$ & 5 & $\begin{array}{c}\text { Curandero o } \\
\text { partera }\end{array}$ & 4 & & & & \\
\hline $56-65$ & 6 & $61-70$ & 4 & Comerciante & 1 & & & & \\
\hline $66-75$ & 5 & & & & & & & & \\
\hline $76-85$ & 5 & & & & & & & & \\
\hline $86-90$ & 1 & & & & & & & & \\
\hline
\end{tabular}

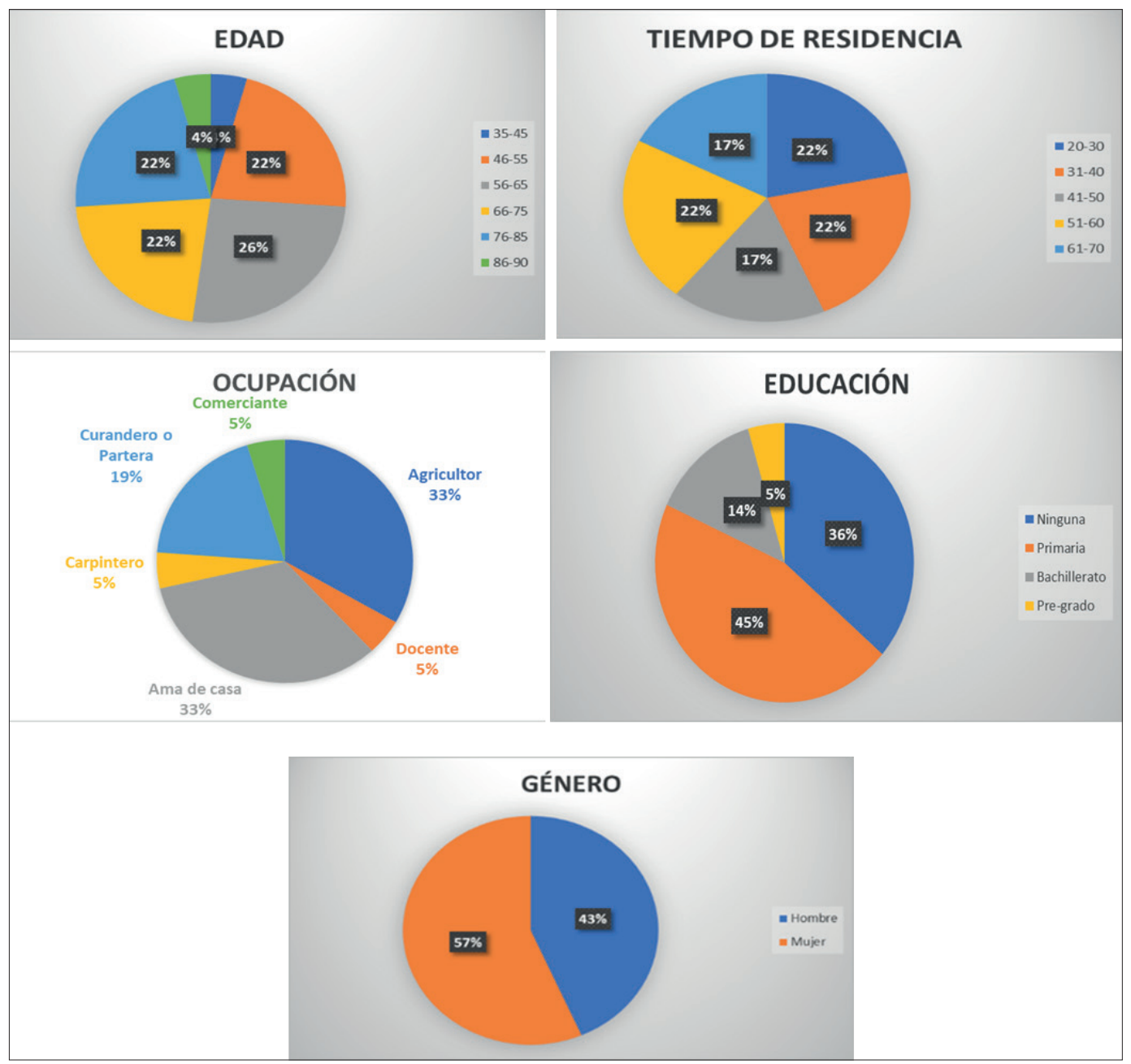

Figura 14. Datos de los informantes del recinto Jauneche. 
Rev. Cient. Cien. Nat. Ambien. 15(2):278-288

Diciembre 2021
Valdiviezo \& Bonifaz • Etnobotánica de las plantas más utilizadas en el Recinto Jauneche, Cantón Palenque, Provincia Los Ríos, Ecuador

\section{Discusión}

En el presente trabajo se encontraron un total de 84 plantas útiles en el Recinto Jauneche, información recolectada por medio de entrevistas y encuestas, los usos etnobotánicos comunes que se mencionaron fueron: medicinal, alimenticio, construcción y rudimentario (utensilios caseros), aunque no se mencionaron las plantas tóxicas del lugar como lo reporta Bonifaz (1997), donde se registraron 26 plantas tóxicas como Aristolochia odoratissima y Mormodica charantia, las mismas que en el presente trabajo son mencionadas como plantas medicinales.

La clasificación etnobotánica de las 84 plantas útiles que se registraron en este trabajo se distribuyeron en cuatro usos dando como resultado 48 especies aplicadas en medicina, 22 plantas utilizadas como alimenticias, 11 en construcción y 3 en rudimentaria (utensilios caseros), comparados estos datos con los reportados para la Cuenca media del Río Guayas en los sectores de Mocache y Palenque según los resultados de Garcia, Suatunce \& Torres (2008) reportan un total de 262 especies útiles distribuidas en 21 usos de los cuales el mayor uso es para medicina y alimento, con 90 y 104 plantas respectivamente, indica que el recinto Jauneche posee infromación etnobotánica en un 32\%, siendo importante, pues el uso y la información ancestral todavia se mantiene en este sector.

Estudios que fueron realizados en la provincia de Los Ríos muestran que el número de plantas útiles usadas como medicinales difiere en algunos cantones, un estudio enfocado en plantas medicinales en el Cantón Quevedo por Zambrano, Buenaño \& Jiménez (2015) muestra un total de 43 plantas medicinales, mientras que en el cantón Ventanas, Paredes, Buenaño \& Mancera (2015), reportaron un total de 33 plantas con uso medicinal, en el presente trabajo que se realizó en el Recinto Jauneche del cantón Palenque se encontraron un total de 47 plantas que son usadas como medicina. Siendo, necesario relevar que el Recinto Jauneche es una extensión mucho menor al Cantón Quevedo y Ventanas, sin embargo, la información etnobotánica de plantas medicinales es mayor que la de dichos Cantones.

Erygium foetidum conocida como culantro de monte es una de las plantas útiles que su uso varía o tiene más de una utilidad, en el presente trabajo de acuerdo a la información recolectada fue mencionada como planta alimenticia (condimento), mientras que Rosero, Zambrano, García \& Viracocha (2020) clasifican a esta especie con 3 usos, alimenticia, medicinal y ornamental según datos obtenidos de los habitantes de la parroquia San Antonio de Padua cantón Quinsaloma, demostrando más de una aplicación en la misma Provincia.

La clasificación etnobotánica ha tenido mayores resultados en las plantas medicinales, así como existen diferencias en usos existen semejanzas, Velásquez et al. (2016) mencionan a Plantago major y Solanum nigrescens como plantas usadas en lavado de heridas, de la misma manera Lalama, Montes \& Zaldumbide (2016) reportan a Scoparia dulcis con el mismo uso, las mismas especies en Jauneche fueron mencionadas con esta utilidad lo que demuestra similitud de usos en los diferentes cantones de la Provincia de los Rios.

Las partes más utilizadas en este estudio demostraron ser las hojas, frutos, tronco y toda la planta, este resultado coincide con Cuchisacagua (2019), Sánchez \& Torres (2020) y Rodríguez, Narváez \& Tuz (2020), donde se obtuvieron resultados similares señalando las hojas como la parte de las plantas con mayor utilidad.

La clasificación taxonómica de este trabajo dio como resultado que las familias Lamiaceae, Asteraceae, Fabaceae, Solanaceae, Acanthaceae y Malvaceae obtuvieron el mayor uso por especies de plantas útiles. Otros estudios demuestran resultados similares como Castellanos (2011), que menciona Asteraceae, Lamiaceae y Solanaceae como familias con el mayor número de especies útiles. Por otro lado, Huamantupa, Sandoval, Betancourt, Sarmiento, \& Telles (2010), mencionan a las familias Fabaceae, Malvaceae, Lamiaceae con mayor número de especies con uso etnobotánico, demostrando coincidencia en trabajos similares.

\section{Conclusiones}

En el Recinto Jauneche utilizan las plantas en cuatro categorías de usos: medicinal, alimenticia, construcción y rudimentaria (utensilios caseros), donde el mayor uso es de plantas medicinales con $57 \%$, mientras que alimenticias obtuvieron $26 \%$, construcción $13 \%$ y $4 \%$ rudimentarias (utensilios caseros).

El registro de 84 especies de plantas útiles en el Recinto Jauneche puede ser considerado alto, debido a que se registró información con una muestra de 23 personas solamente, indicando que el conocimiento etnobotánico está presente en los habitantes del recinto, aduciendo este factor a la relación cercana que mantienen con el Bosque de la Estación Biológica Pedro Franco Dávila.

La utilidad de las plantas en el recinto Jauneche es mayor en el uso medicinal, pues el conocimiento ancestral se mantiene, aunque la medicina convencional está presente mediante el subcentro de salud existente.

Las plantas útiles en el recinto Jauneche corresponden a 37 familias y 61 géneros y 84 especies_identificadas; siendo Lamiaceae, Asteraceae, Acanthaceae, Fabaceae, Malvaceae y Solanaceae las familias taxonómicas con más plantas útiles en el recinto. 
Rev. Cient. Cien. Nat. Ambien. 15(2):278-288 Diciembre 2021
Valdiviezo \& Bonifaz • Etnobotánica de las plantas más utilizadas en el Recinto Jauneche, Cantón Palenque, Provincia Los Ríos, Ecuador
Plantago major fue la especie que 18 de los 23 informantes mencionaron que la utilizaban como planta medicinal para desinfectar heridas siendo la planta útil con mayor frecuencia de uso.

Las especies de menor uso son importantes debido a que en este grupo se encuentran plantas medicinales como la Aphelandra attenuata usada para baños medicinales por los curanderos que viven en el lugar.

Del grupo de informantes la información recolectada fue principalmente de agricultores y amas de casas debido a la disponibilidad de tiempo que poseen estos dos grupos

En la zona céntrica del recinto Jauneche son cuatro las personas que se dedican a oficios como parteras o curanderos, aunque el número no es representativo demostraron poseer conocimiento suficiente sobre las plantas útiles del lugar de manera especial las plantas medicinales.

\section{Recomendaciones}

- Ampliar el estudio de plantas útiles en el Recinto Jauneche incluir otras categorías de usos.

- En próximos trabajos se debe tomar en consideración los sectores pertenecientes al recinto Jauneche y el periodo de mayor precipitación, debido a que algunas especies solo se encuentran en época lluviosa.

- Una herramienta que permitirá un mejor estudio es la entrevista directa con al menos 5 personas que tengan conocimiento de plantas útiles.

- Realizar un estudio sólo de plantas medicinales por la cantidad de especies que fueron reportadas en este uso y aquellas que no fueron identificadas o encontradas.

- Elaborar una ficha de las plantas útiles del Recinto Jauneche con información completa que incluya fotografías de las plantas.

- Realizar un estudio fitoquímico de las plantas medicinales poco comunes de manera particular la especie Euphorbia hirta.

- Realizar un estudio del impacto de la agricultura en las plantas útiles y su relación con el bosque protector.

\section{Referencias}

Bonifaz, C. 1997. Plantas tóxicas de la Provincia de los Ríos, litoral ecuatoriano. En M. Ríos, \& H. Borgtoft Pedersen, Uso y Manejo de Recursos Vegetales (págs. 339-346). Quito: Abya-Yala.

Castellanos, L. 2011. Conocimiento etnobotánico, patrones de uso y manejo de plantas útiles en la Cuenca del río Cane-Iguaque (Boyaca-Colombia); una aproximación desde los sistemas de uso de la Biodiversidad. Ambiente \& Sociedade, 45-75.

Cerón, C. 2002. Aportes a la flora útil de Cerro Blanco, Guayas-Ecuador. Cinchonia, 3, 17-25.

Cuchisacagua, R. 2019. Estudio etnobotánico de la biodiversidad vegetal en la comunidad Verde Sumaco, provincia de Orellana. (Tesis de grado. Ingeniero en Ecoturismo) Dspace. Obtenido de http://dspace.espoch. edu.ec/handle/123456789/10339

De la Torre, L., Navarrete, H., Macía, M., y Balslev, H. 2008. Introducción. En L. de la Torre, H. Navarrete, P. Muriel, M. Macía, \& H. Balslev, Enciclopedia de las Plantas Útiles del Ecuador (págs. 1-3). Quito \& Aarhus: Herbario QCA de la Escuela de Ciencias Biologicas de la Pontifica Universidad Católica del Ecuador \& Herbario AAU del Departamento de Ciencias Biológicas de la Universidad de Aarhus.

De la Torre, L., Navarrete, H., Macía, M. y Balslev, H. 2008. Resultados. En L. De la Torre, H. Navarrete, P. Muriel, M. Macia, \& H. Balslev, ENCICLOPEDIA DE LAS PLANTAS ÚTILES DEL ECUADOR (págs. 8-12). Quito \& Aarhus: Herbario QCA de la Escuela de Ciencias Biológicas de la Pontificia Universidad Catóica del Ecuador \& Herbario AAU del Departamento de Ciencias Biológicas de la Universidad de Aarhus.

Garcia, L., Suatunce, P., y Torres, E. 2008. Plantas útiles en los sistemas agroforestales tradicionales del litoral ecuatoriano. Ciencia y tecnología, 65-71.

Huamantupa, I., Sandoval, V., Betancourt, L., Sarmiento, M. y Telles, F. 2010. Plantas útiles frecuentemente utilizadas en el litoral costero del Ecuador. $Q^{\prime} U \tilde{N} A, 28-38$.

Lalama, J., Montes, S. y Zaldumbide, M. 2016. Etnobotánica de las plantas medicinales del cantón Tena, para contribuir al conocimiento, conservación y valoración de la diversidad vegetal de la región amazónica. Dominio de las Ciencias, 26-48.

Paredes, D., Buenaño, M. y Mancera, N. 2015. Uso de plantas medicinales en la comundiad San Jacinto del Cantón Ventanas, Los Ríos-Ecuador. Revista U.D.C.A Actualidad \& divulgación científica, 39-50.

Peña, C., Guerrero, M., Torre, J. y Sánchez, G. 2020. Cultura ancestral florística de la comunidad kichwa singue central, en la amazonía ecuatoriana. Enlace Universitario, 115-124.

Petran, M., Dragos, D. y Gilca, M. 2020. Historical ethnobotanical review of medicinal plants used to treat children disases in Rumania (1860s-1970s). Journal of Ethnobiology and Ethnomedicine, 1-33.

Polanco, D., Vernaza, D. y Burbano, C. 2018. Potencialidades y debilidades de las plantas nativas de uso alimenticio. Tsafiqui, 2-19.

Ríos, M., Koziol, M., Borgtoft, H. y Granda, G. 2007. La Etnobotánica en el Ecuador: Síntesis, Retos y Perspectivas. Quito: Corporación Sociedad para la Investigación de la Biodiversidad Ecuatoriana (SIMBOE).

Rodríguez, M. R., Narváez, K. y Tuz, J. 2020. Conocimientos sobre plantas rituales utilizadas por yerbateras de los mercados de Quito, Ecuador: aportes sobre su estado de conservación. Ethnoscientia.

Rosero, C., Zambrano, L., García, K. y Viracocha, L. 2020. Nomenclatura y usos del culantro de monte (Eryngium foetidum L.) en la comunidad San Antonio de Padua, cantón Quinsaloma, Provincia de Los Ríos-Ecuador. Boletín Latinoamericano y del Caribe de plantas medicinales y arómaticas, 334-343.

Sánchez, J., y Torres, L. 2020. Educación. Etnobotánica y rescate de saberes ancestrales en el Ecuador. ESPACIOS, 158-170.

Velasquez, G., Moreira, W., Caicedo, C., Preciado, O., Jaramillo, T., Iturralde, G. y Aizaga, G. 2016. El uso de la etnobotánica como cura de eruptivas de la infacia por la etnia Chachi. Esmeraldas. Ecuador. Talentos, 1-10. 
Rev. Cient. Cien. Nat. Ambien. 15(2):278-288

Diciembre 2021

ISSN: $2773-7772$
Valdiviezo \& Bonifaz • Etnobotánica de las plantas

más utilizadas en el Recinto Jauneche, Cantón

Palenque, Provincia Los Ríos, Ecuador

Yánez, P. 2017. Plantas frecuentemente utilizadas en zonas rurales de la Región Amazónica centro occidental de Ecuador. INNOVA, 9-21.

Zambrano, L., Buenaño, M., Néstor, M. y Jiménez, E. 2015. Estudio etnobotanico de plantas medicinales utilizadas por los habitantes del área rural de la parroquia San Carlos, Quevedo, Ecuador. Revista Universidad y Salud, 97-111. 